\title{
DEVELOPMENT OF ENTREPRENEURIAL ACTIVITY, LABOR MARKET AND MODERN CHALLENGES OF HIGHER EDUCATION
}

\section{GIVI BEDIANASHVILI}

Doctor of Economic Sciences, Professor

European University, Institute for Research of Economic and

Social Problems of Globalization, Associate Professor,

Ivane Javakhishvili Tbilisi State University,

Academician of the Georgian Academy of Economic Sciences, Georgia

g.bedianashvili@eu.edu.ge

\section{EKATERINE NATSVLISHVILI}

Doctor of Philosophy, Associate Professor

European University, Georgia

natsvlishvili.ekaterine@eu.edu.ge

\author{
QUJI BICHIA \\ Doctor of Economics, Associate Professor \\ European University, Institute for Research of Economic \\ and Social Problems of Globalization, Georgia \\ q.bichia@iset.ge
}

Abstract. Entrepreneurial activity has always been a major factor in the socio-economic development of any country in the world. This is especially evident in the discussion of the problems of economic growth, when the efficiency of the functioning of the entrepreneurial sphere within the country clearly determines both the rates of economic growth and the state of the social sphere and the well-being of the people.

It is noteworthy that in modern conditions, against the background of the high importance of the formation of the knowledge economy, the innovative aspect of entrepreneurial activity in the context of all business entities is gaining special urgency. The effectiveness of the whole innovation process depends on the effective functioning of knowledge-based entrepreneurship.

The aim of the research is to identify the requirements of the entrepreneurial field for human capital skills, to identify the imbalances in the labor market, to determine the compliance of the skills required for the teaching and learning activities of high schools, to identify the existing challenges and to formulate separate recommendations.

The research process used the methods of induction, deduction, qualitative, quantitative, comparison, statistical analysis, benchmarking. The processing of existing literature, retrieval of statistical data (including with the direct assistance of the National Statistics Office of Georgia), automatic download of data (so-called scraping) from various employment sites, conducting surveys and in-depth interviews, sorting and quantitative analysis of retrieved data were included in the own research. The consideration of expert opinions is within the research.

Research has shown that when considering the skills of entrepreneurs, as a starting point, the entrepreneurial activity in a particular country and the requirements for its development should be considered. It should be noted, however, that the labor market may not be able to meet the needs of the entrepreneurial sector (employers).

On the other hand, the perceptions of the entrepreneurial subjects themselves about the required skills are often limited and conditioned by the relatively short-term current perceptions of the modern technologies of the entrepreneurial activity itself.

The research and the analysis of the positive practical experience of the developed countries of the world clearly showed that in this regard, close cooperation with universities in the field of entrepreneurship in the form of entrepreneurship training and short-term courses will be effective. The involvement and support of the state in the development and implementation of innovative entrepreneurial policies would also be important here.

KEYWORDS: ENTREPRENEURIAL ACTIVITY, LABOR MARKET, DEMAND SKILLS, HIGHER EDUCATION CHALLENGES.

For citation: Bedianashvili, G., Bichia, Q., \& Natsvlishvili, E. (2021). Development of Entrepreneurial Activity, Labor Market and Modern Challenges of Higher Education. Globalization and Business. 12, 21-31. (In Georgian). https://doi.org/10.35945/ gb.2021.12.002 


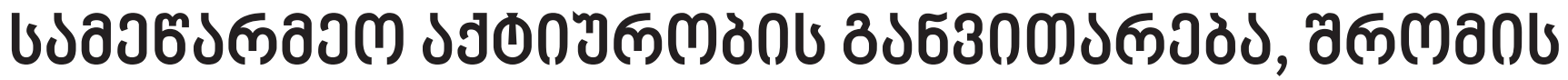

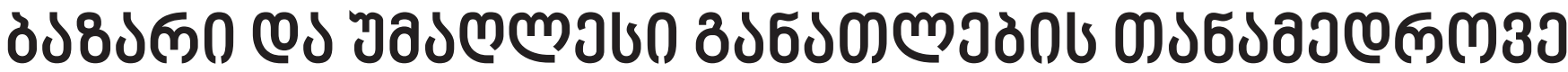 उ১วற63อ3อठ0}

\section{0 ठวழ0১ธงฉ30ल0}

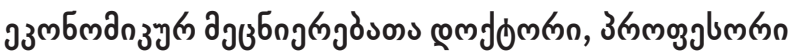

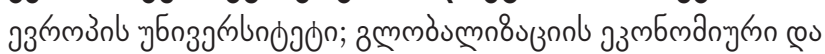

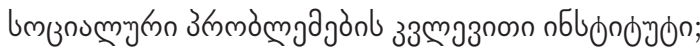

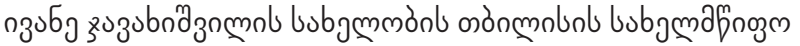

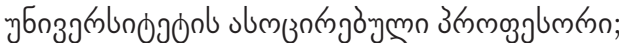

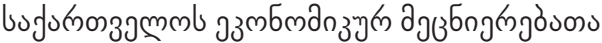

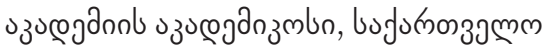

g.bedianashvili@eu.edu.ge

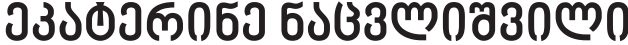

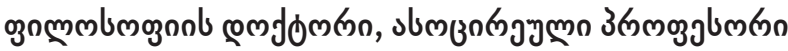

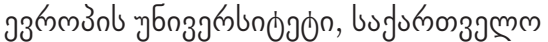

natsvlishvili.ekaterine@eu.edu.ge

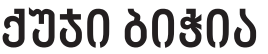

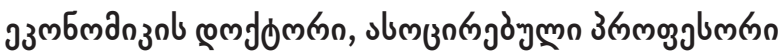

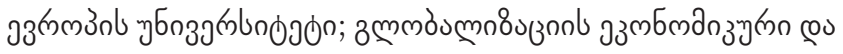

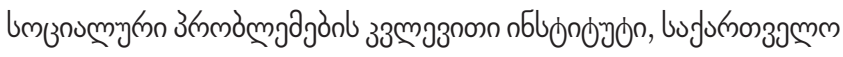 \\ q.bichia@eu.edu.ge
}

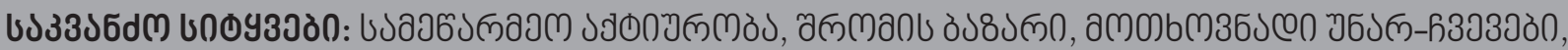

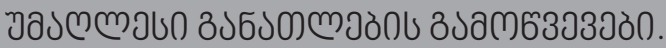

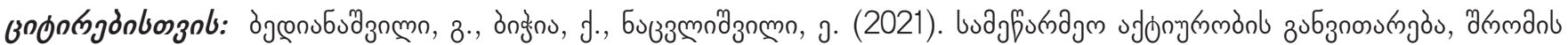

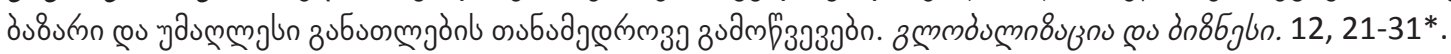

https://doi.org/10.35945/gb.2021.12.002

\section{ซอเงЗ১ตั0}

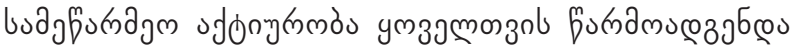

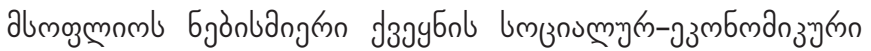

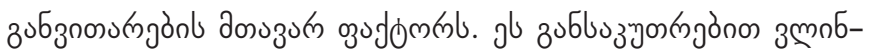

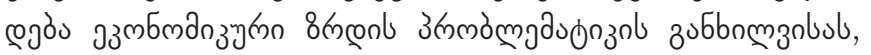

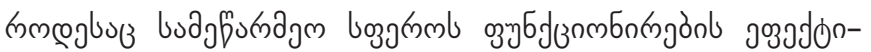

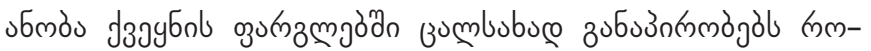

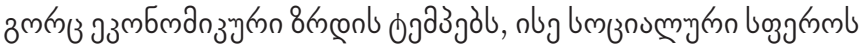

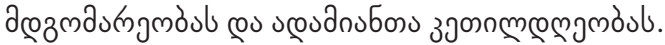

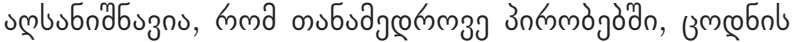

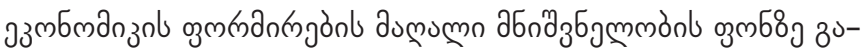

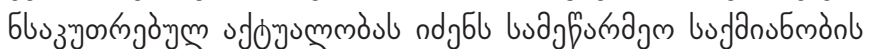

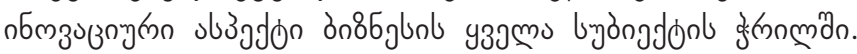

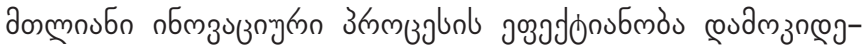

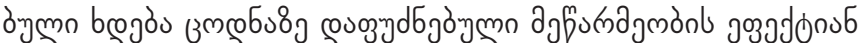

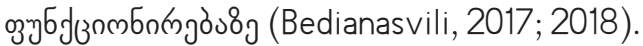

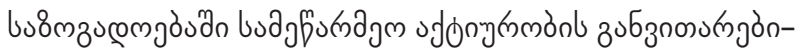

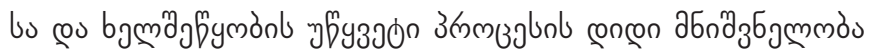

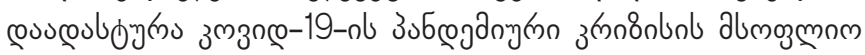

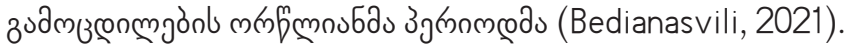

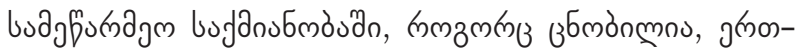

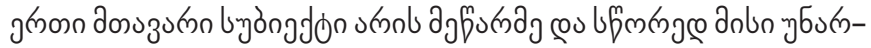

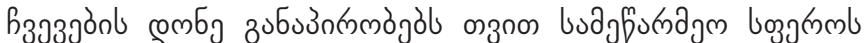

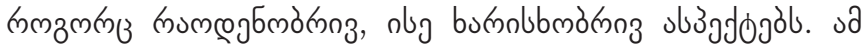

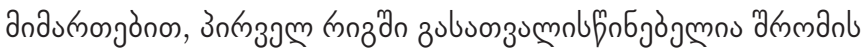

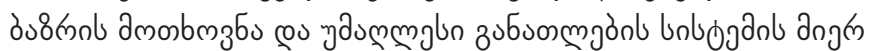

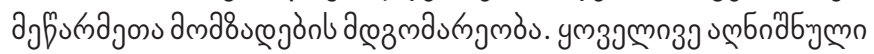

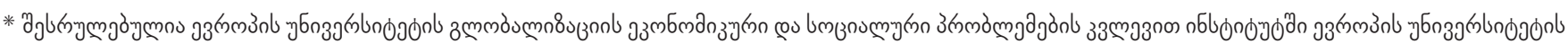

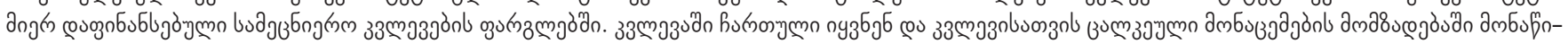

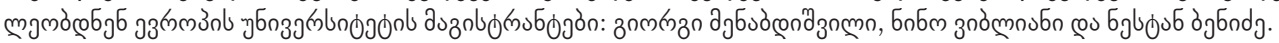




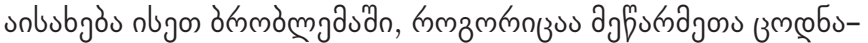

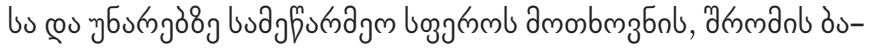

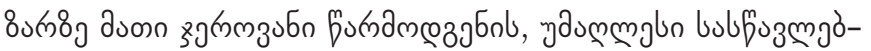

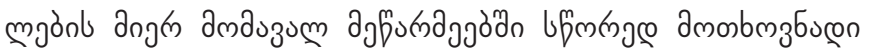

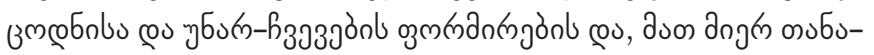

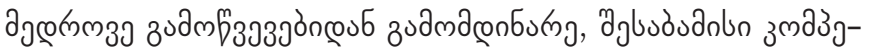

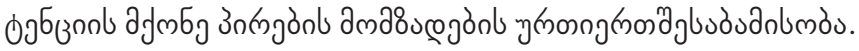

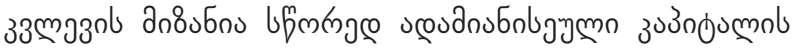
चб

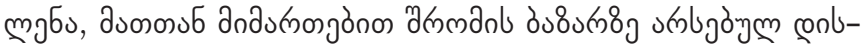

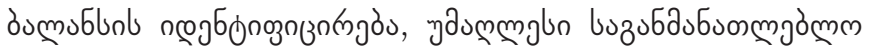

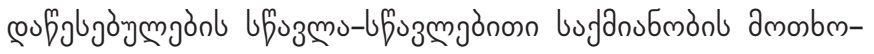

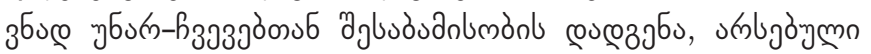
zudm

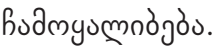

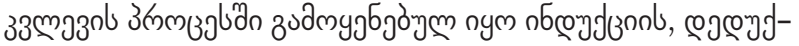

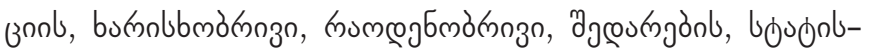

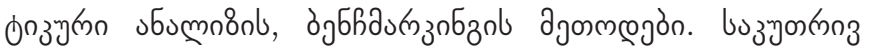

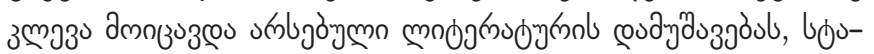

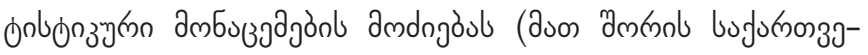
mmb boujobonzol g grmзб

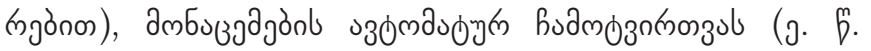

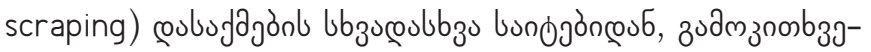
on lou hum

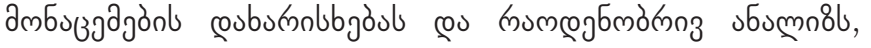
u zumolsfnбogoul.

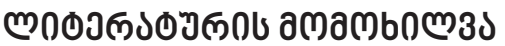

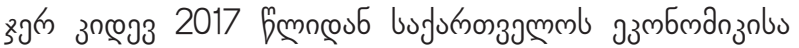

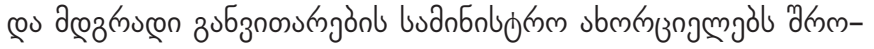

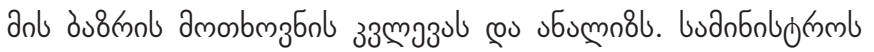

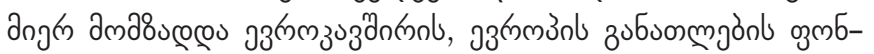

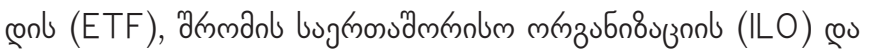

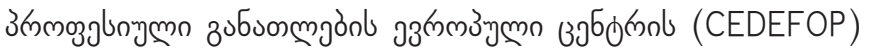

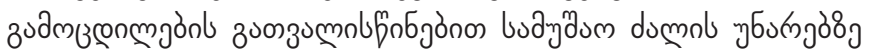

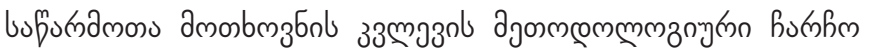

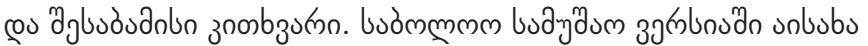
connбogr

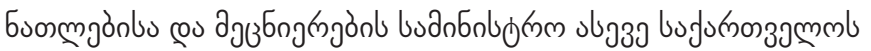

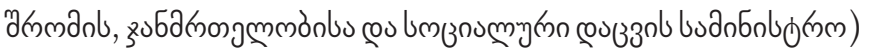
ambuongjòjodon (Georgian Labor Market Analysis, 2017; 2019;

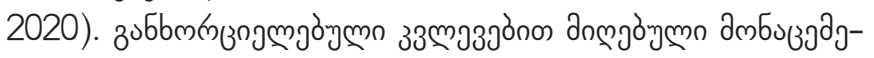

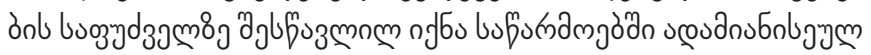

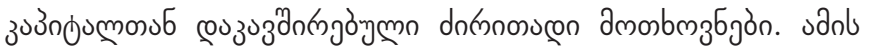

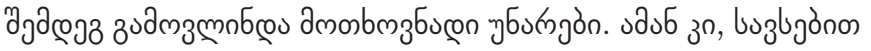

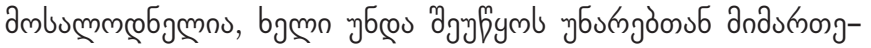

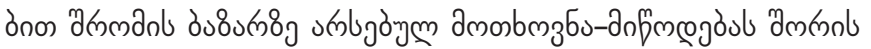

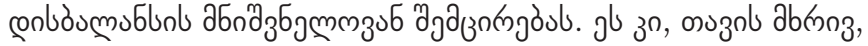

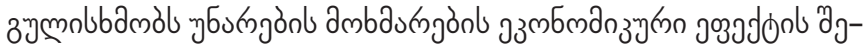

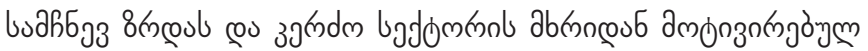

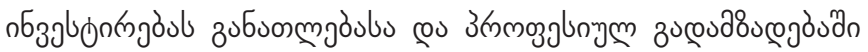
(Georgian Labor Market Analysis, 2020).

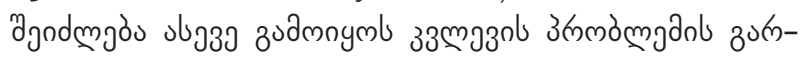

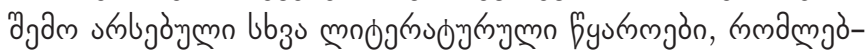

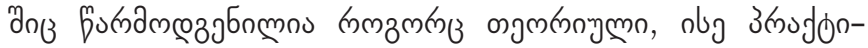
зуymn bubnuonol dubumgòn (nb., duzumnonun, Alterbridge, 2019; Amashukeli, 2021; Chanukvadze, 2021; Gagnidze, 2018; 2019; Khoshtaria, 2018; Employment Problems ..., 2018; Erkomaishvili, 2019; Law of Georgia on Employment ..., 2020; Diakonidze, 2018; Otarashvili, 2021; Strategic Development of Higher Education ..., 2013).

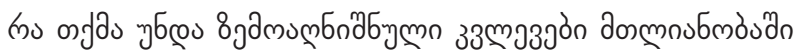

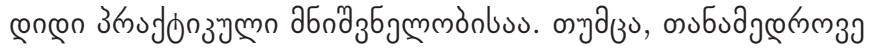

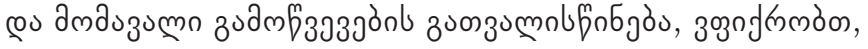

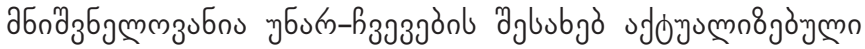

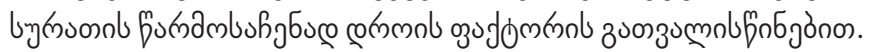

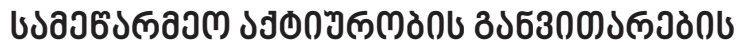

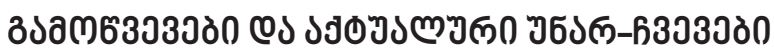

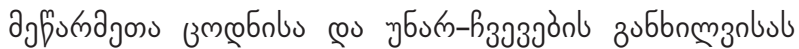

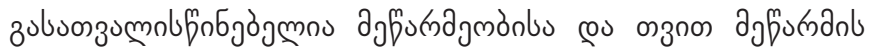

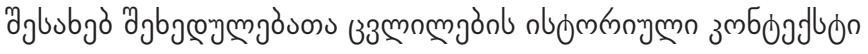

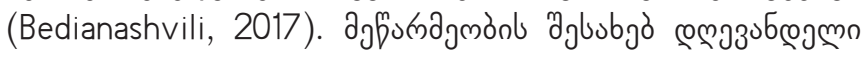

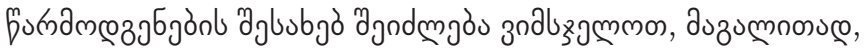

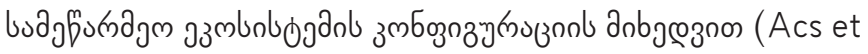

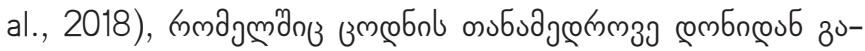

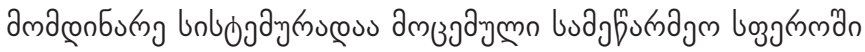

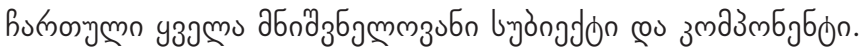

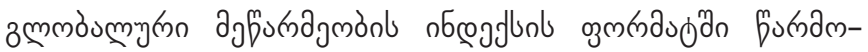

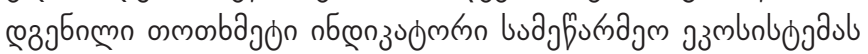

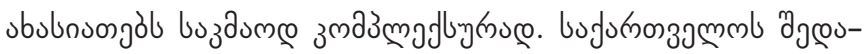

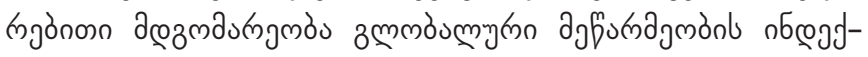

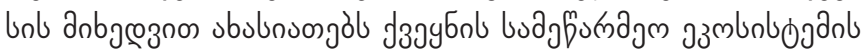

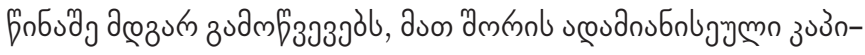
oumonl dnaunon (6ub. 1).

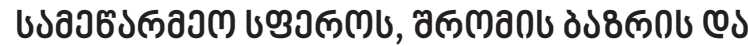

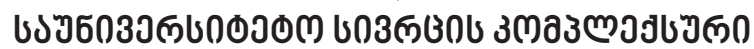

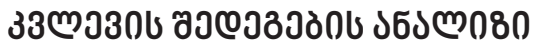

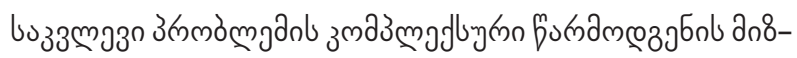

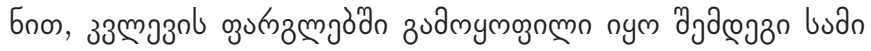

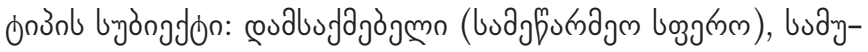

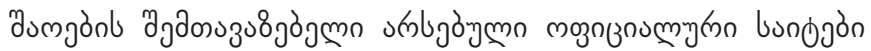




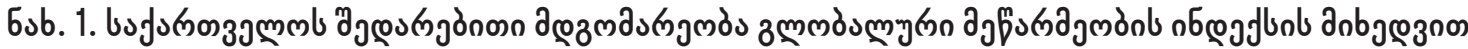

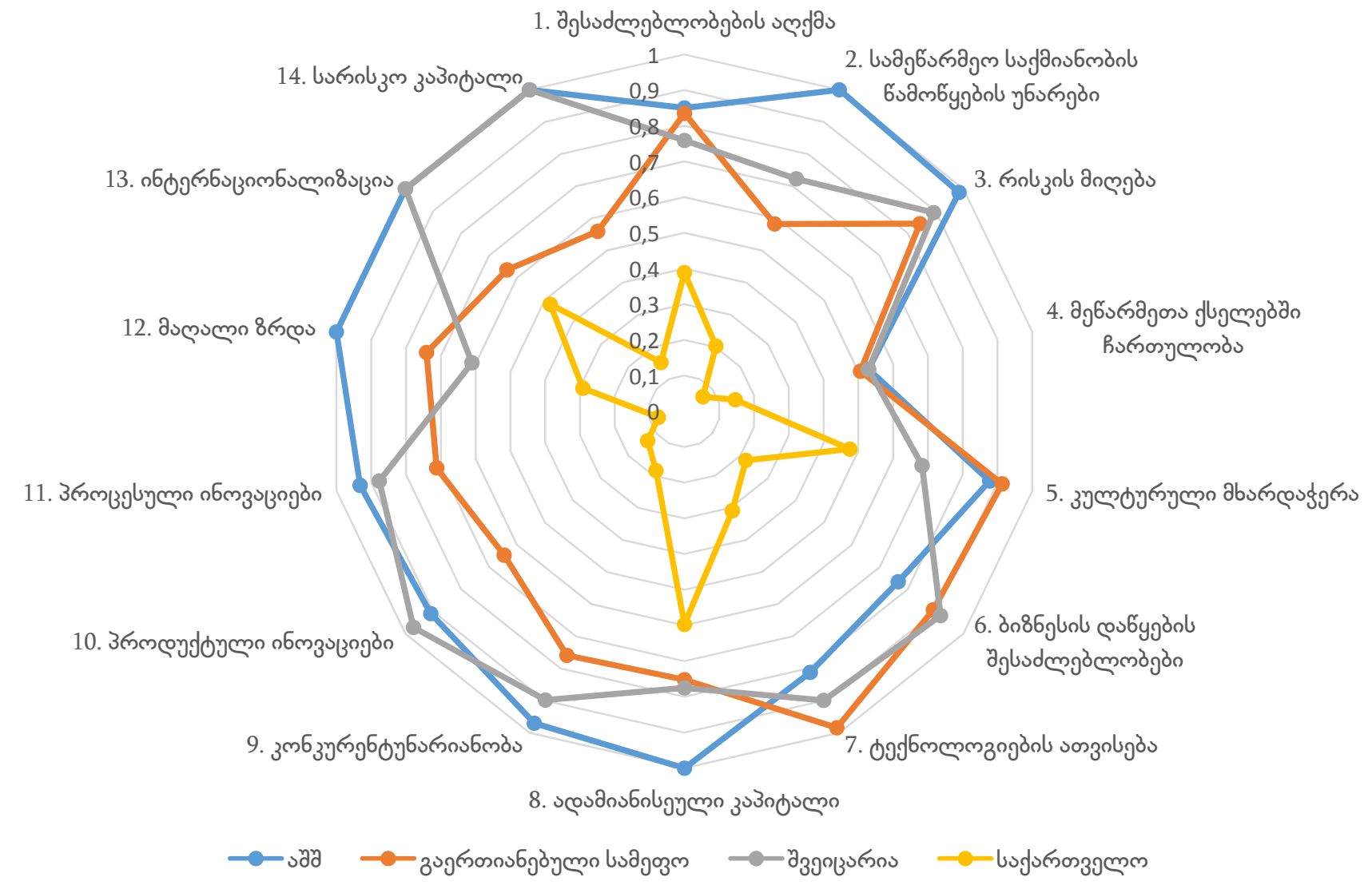

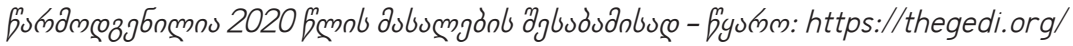

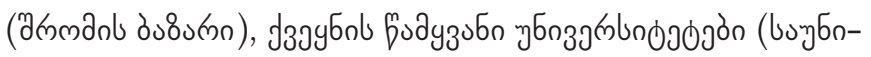

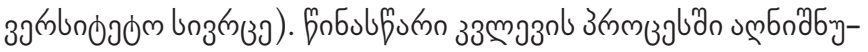

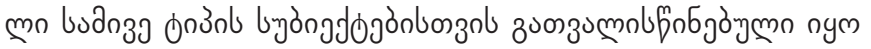

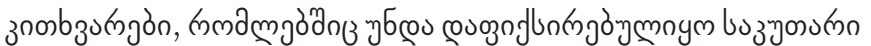

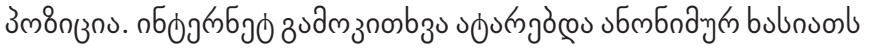

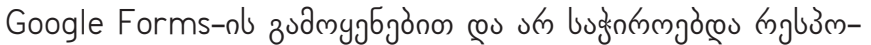

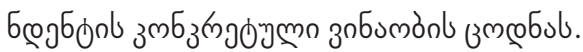

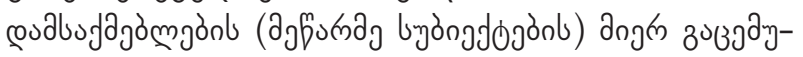

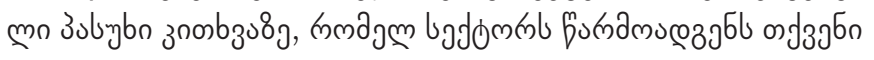

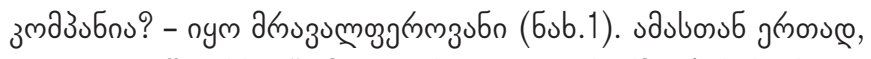

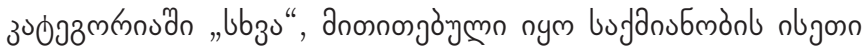

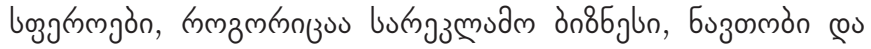

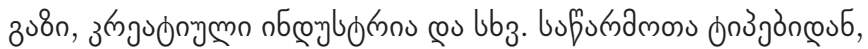

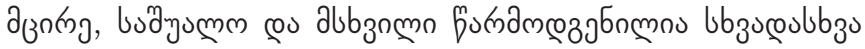
fonmon (6ub. 2).

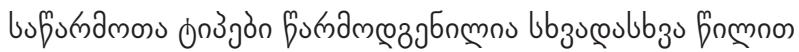

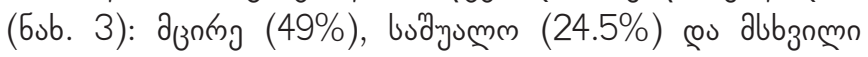
(26.5\%).

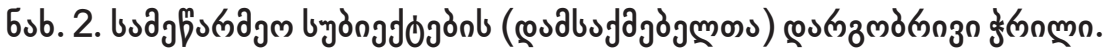

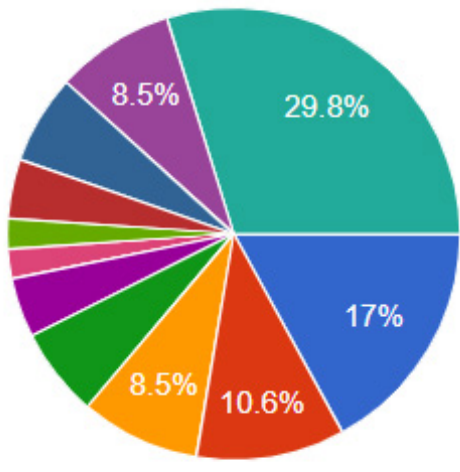

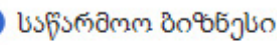

us

usवวృб

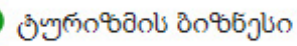

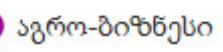

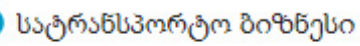

удलॅз

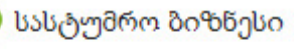

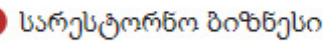

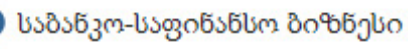

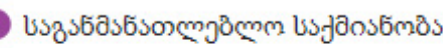

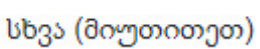




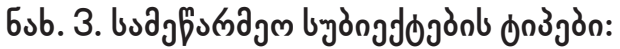

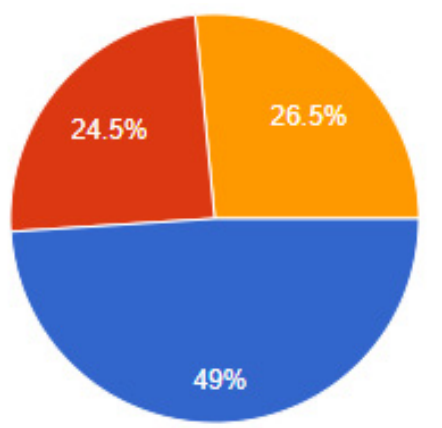

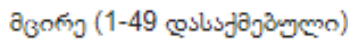

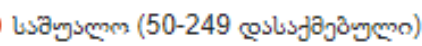

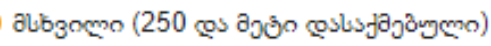

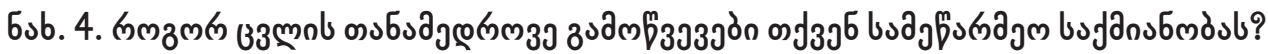

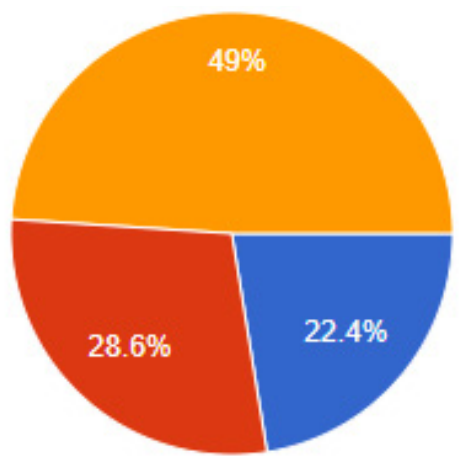

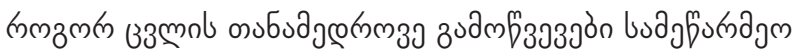

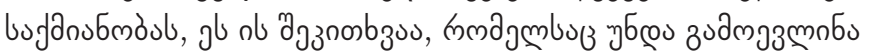

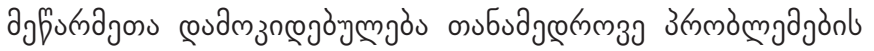

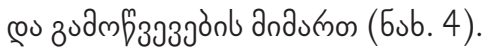

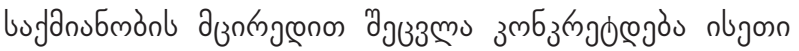

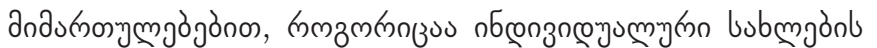

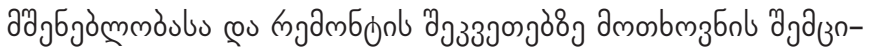

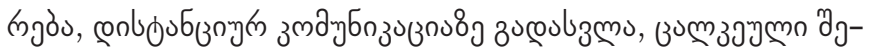

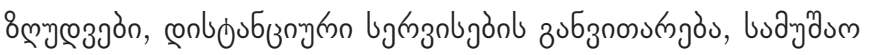

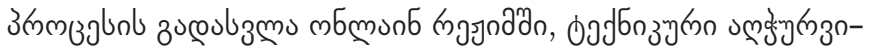

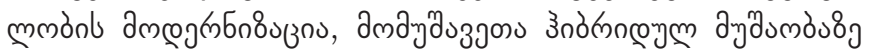

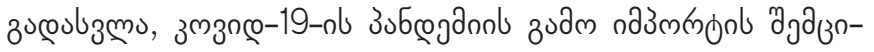

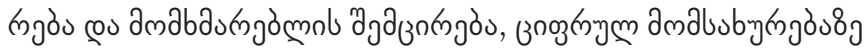

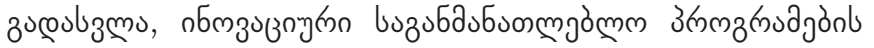

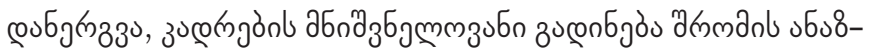

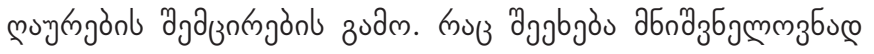

Lisfromesces sismol

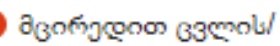

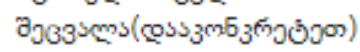

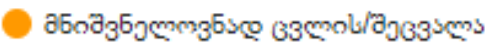

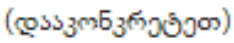

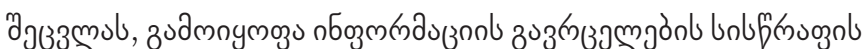

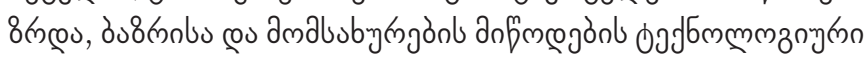

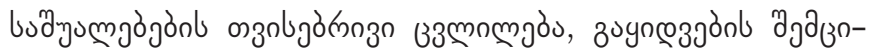

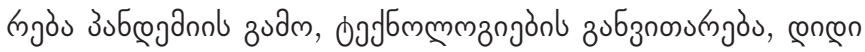

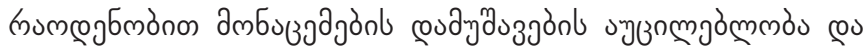

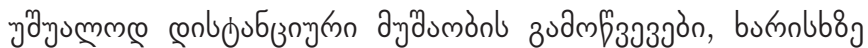

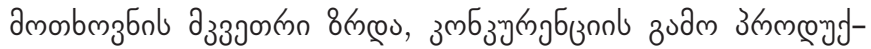

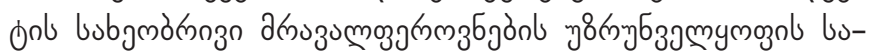

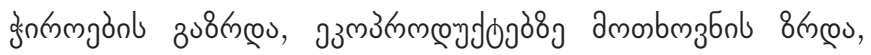

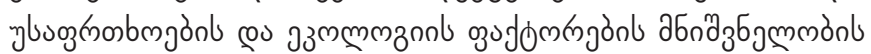

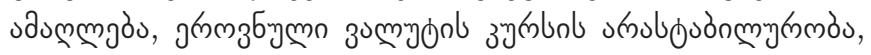

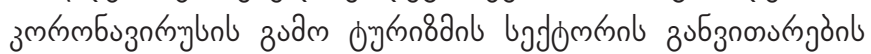

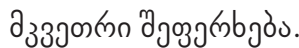

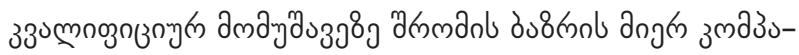

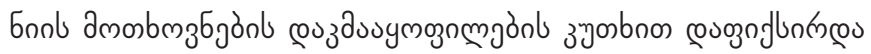

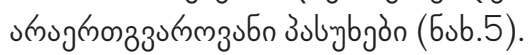

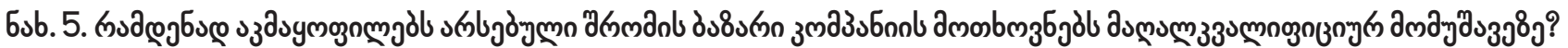

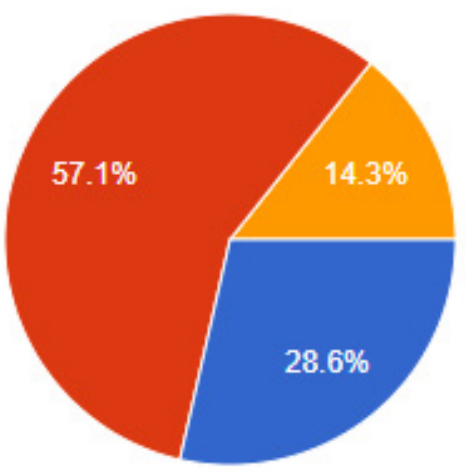

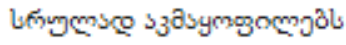

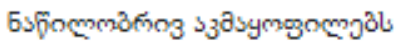

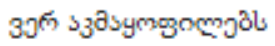




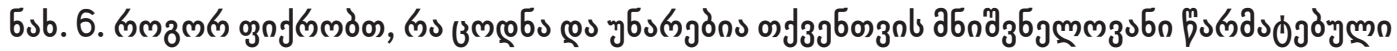

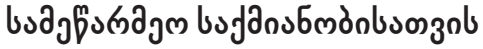

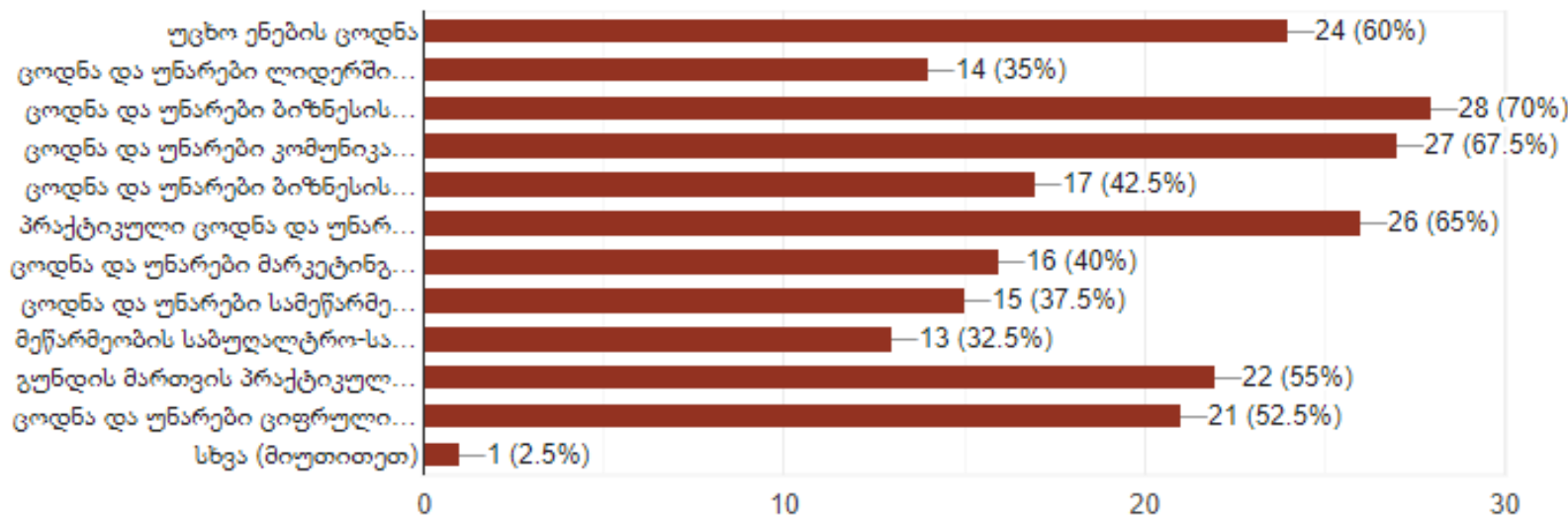

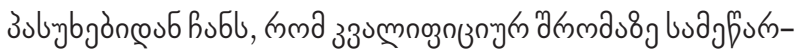

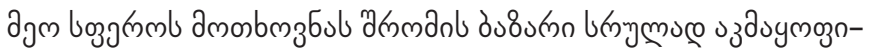

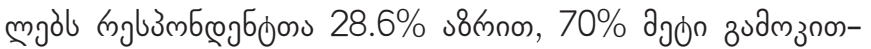
bygmos dmbs8

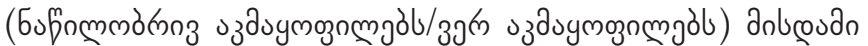

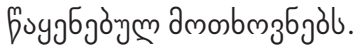

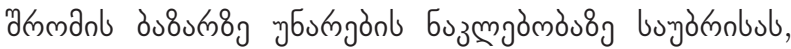

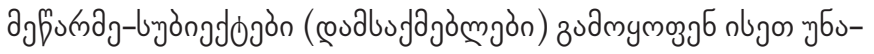

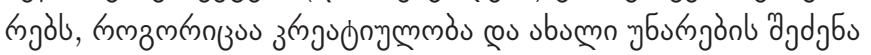

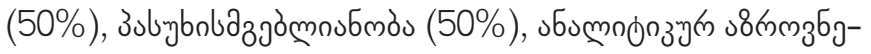

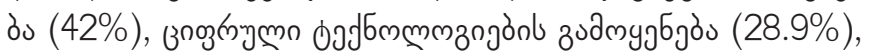

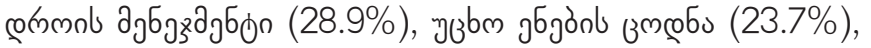

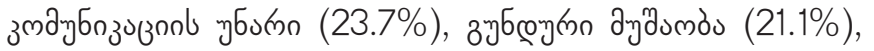

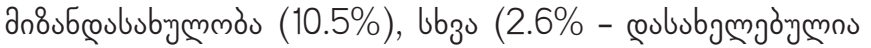

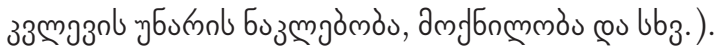

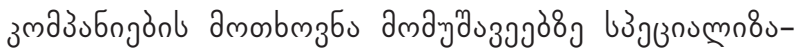

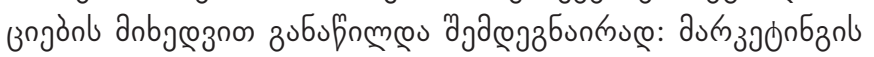

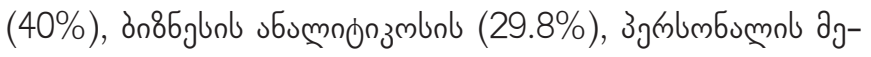

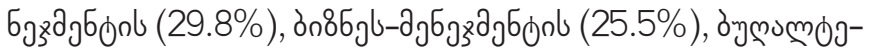

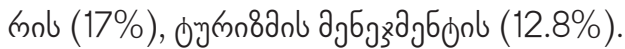

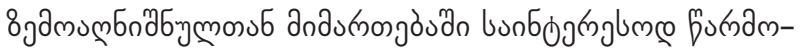

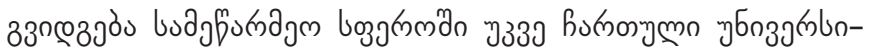

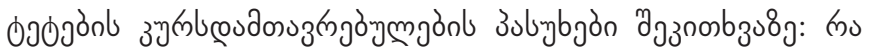

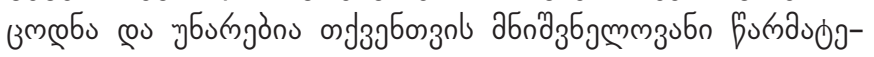

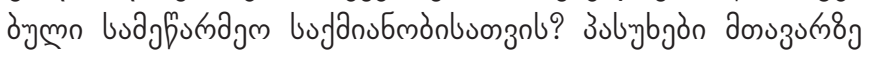

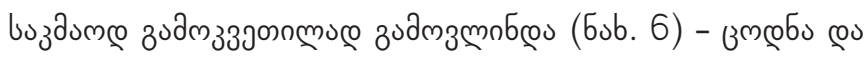

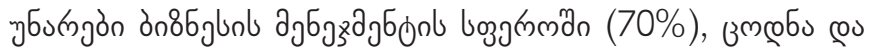

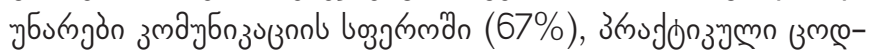

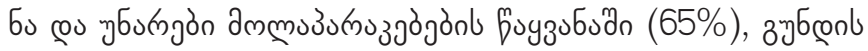

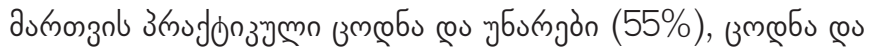

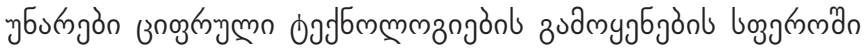

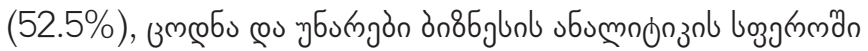

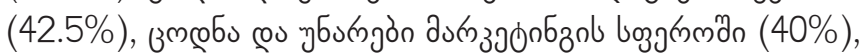

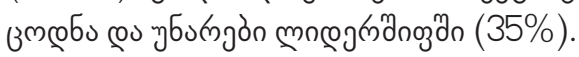

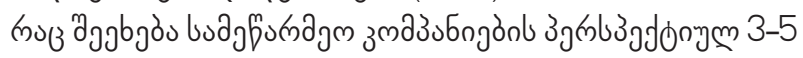

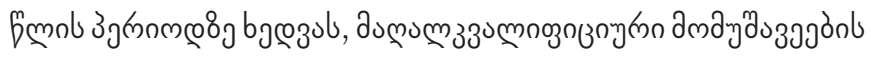

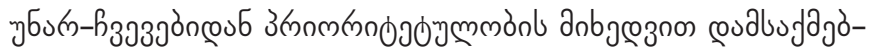

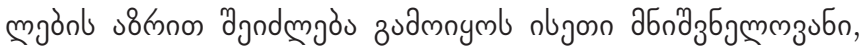

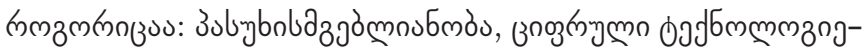

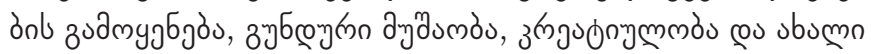

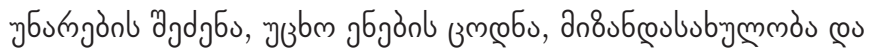

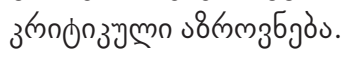

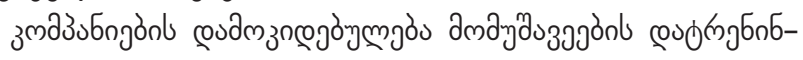

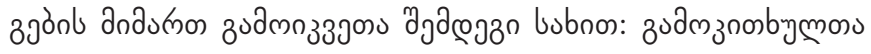

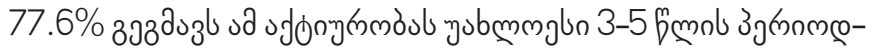

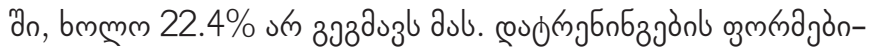

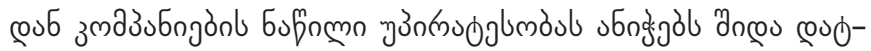

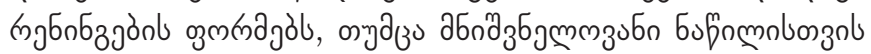

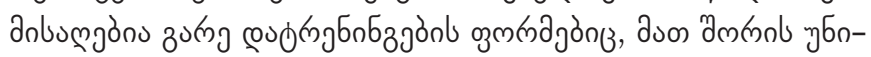

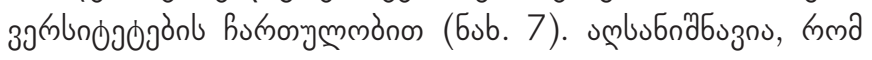

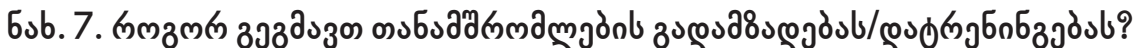

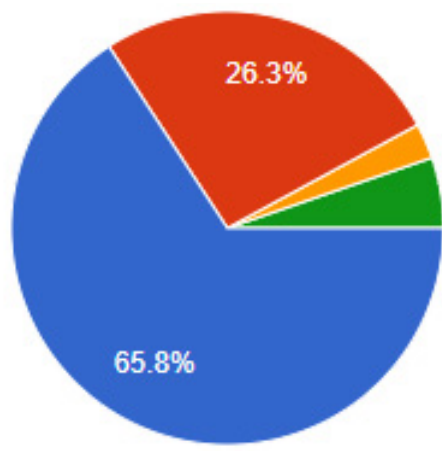

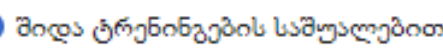

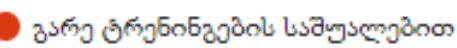

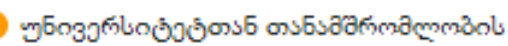
usangsmparoos

lib̧s (anyosnorjos) 


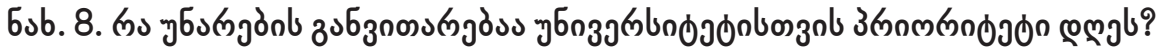

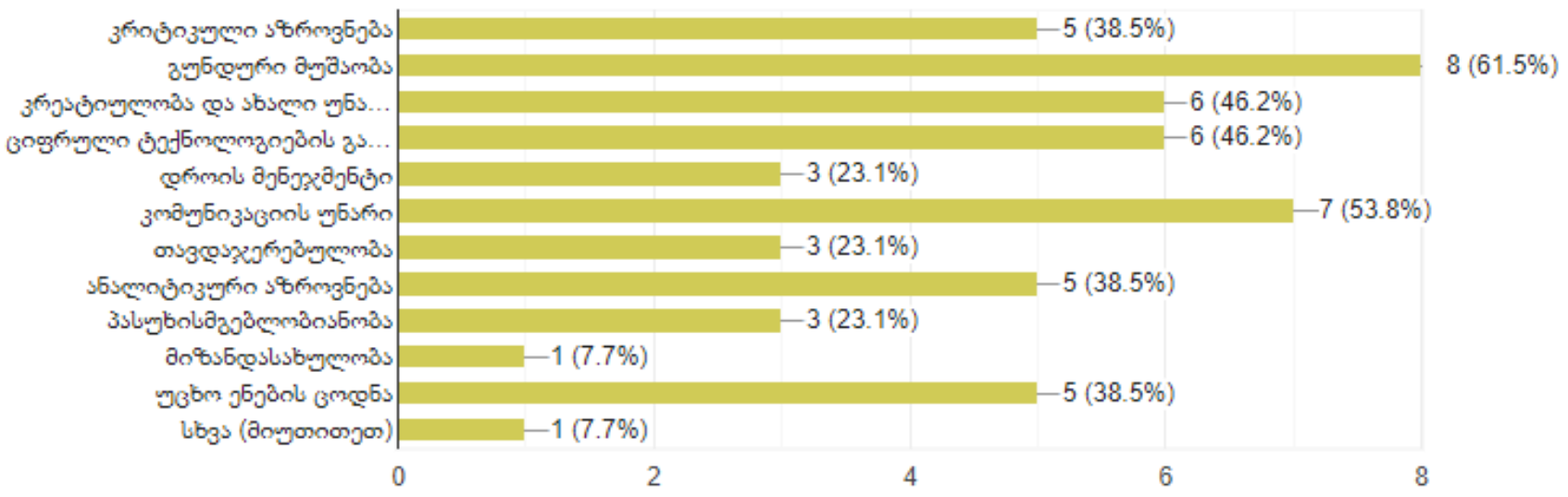

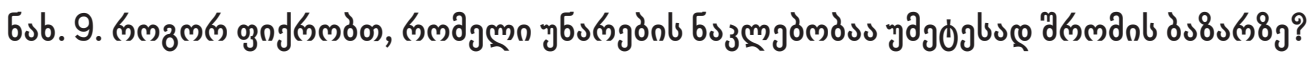

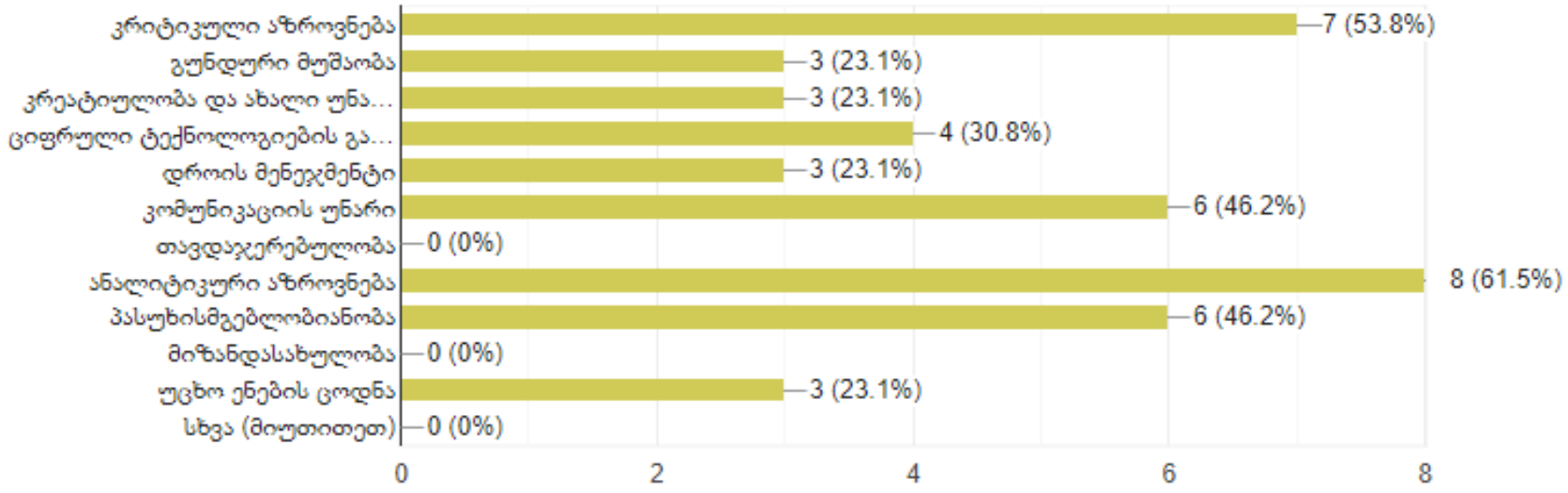

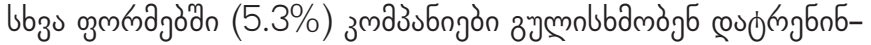
zjonb buanz

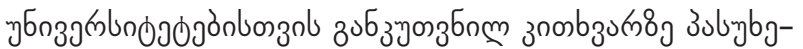

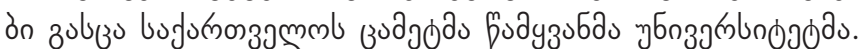

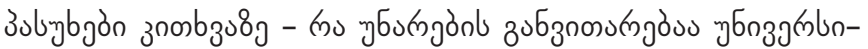

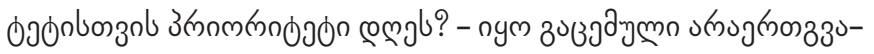
(mмзбом (6ub. 8).

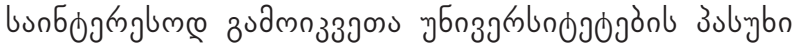

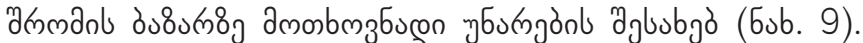

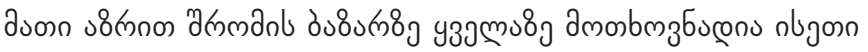

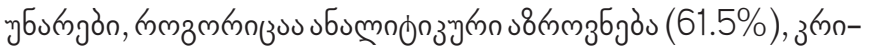

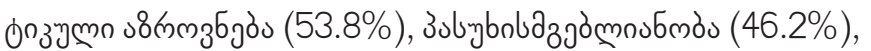

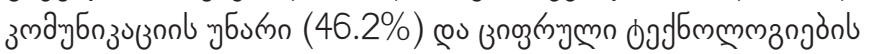

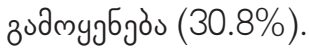

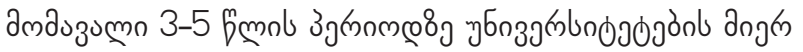

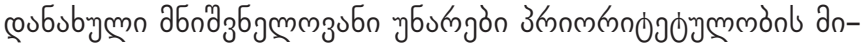

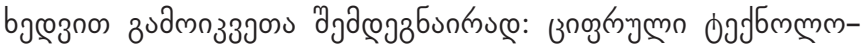

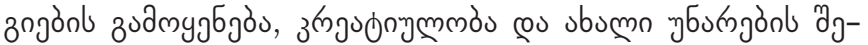

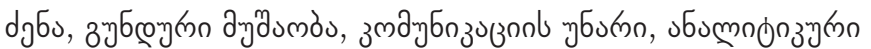

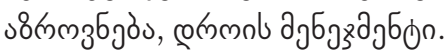

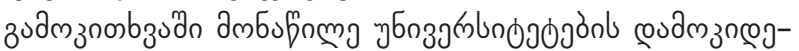

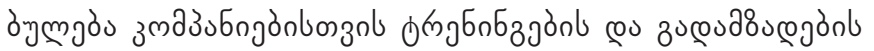
зचn

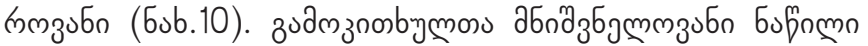

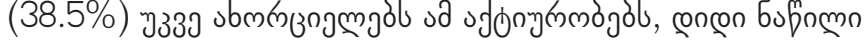

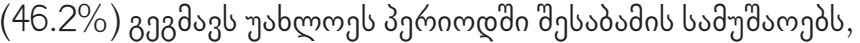

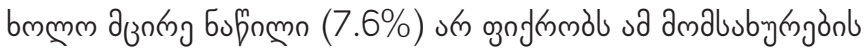

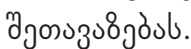

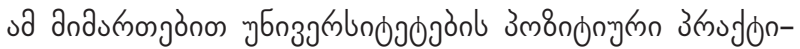

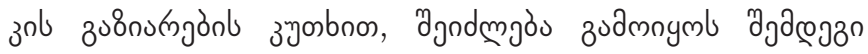

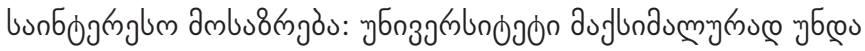

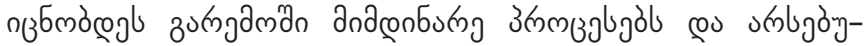

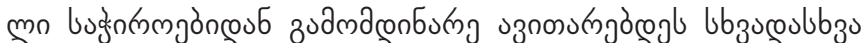

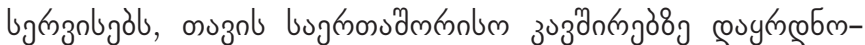

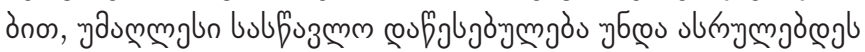

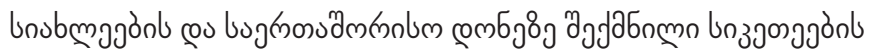

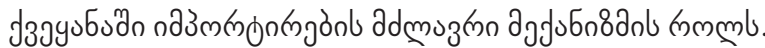

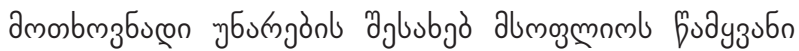

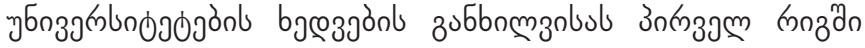

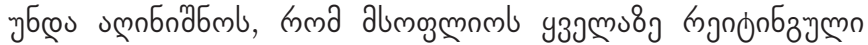
चб

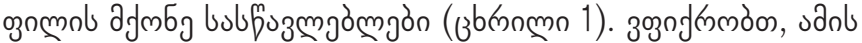

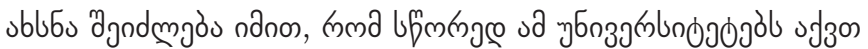

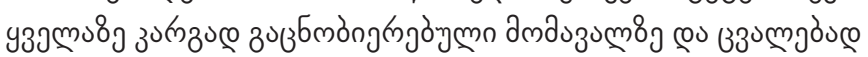

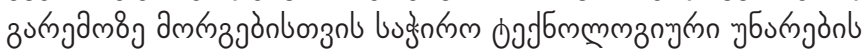

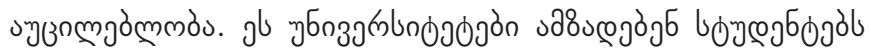

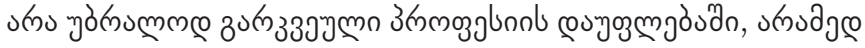

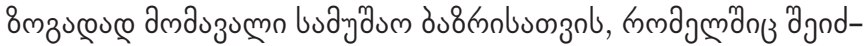




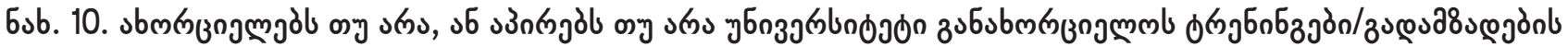

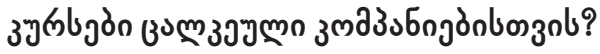
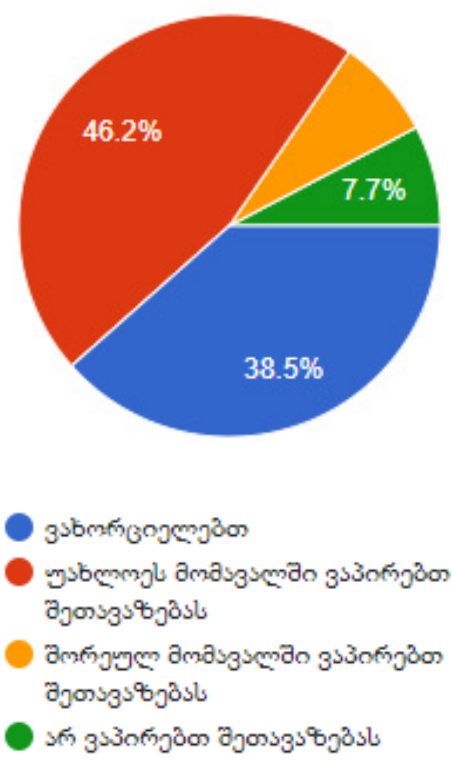

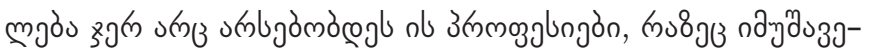
¿j

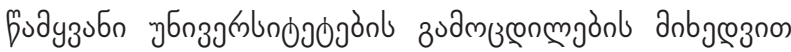

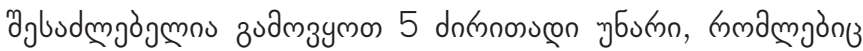

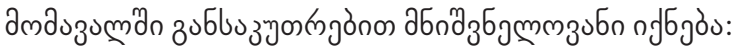

\section{उलз}

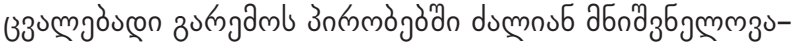

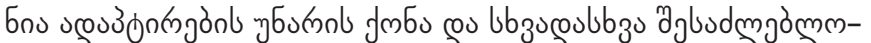

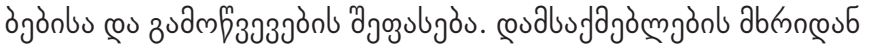

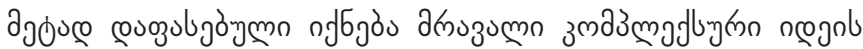

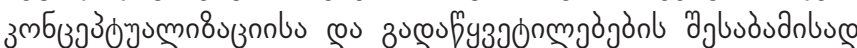
amkrajòn

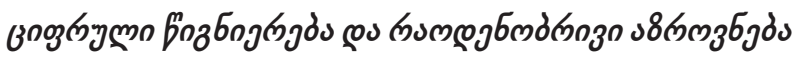

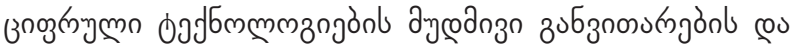

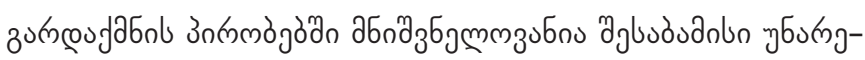

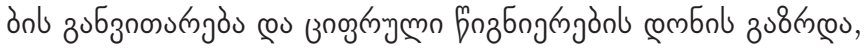

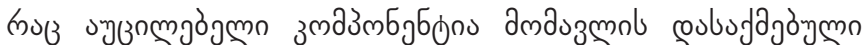

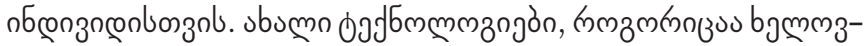

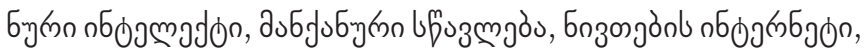

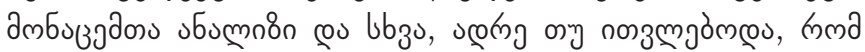

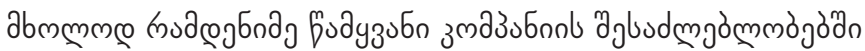

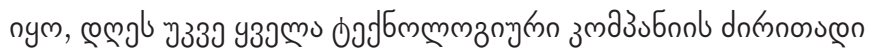
bufanubmònb bufingnos.

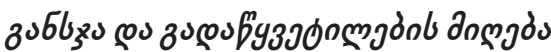

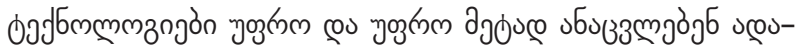

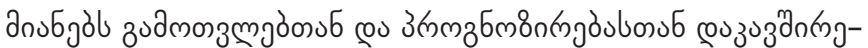

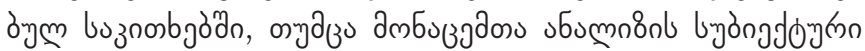
abukng amauzumanno u

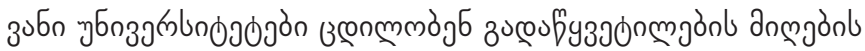

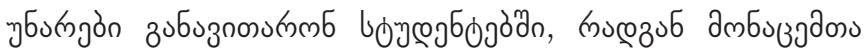

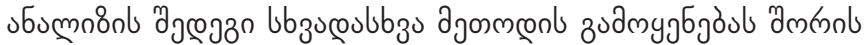

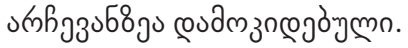

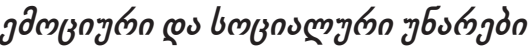

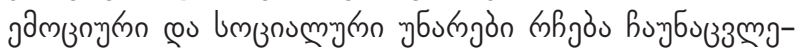

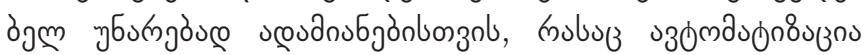

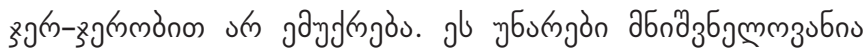

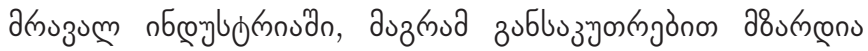

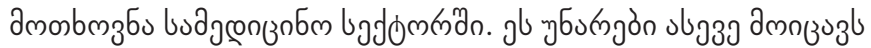

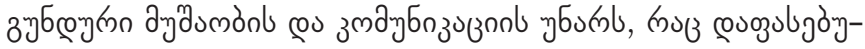

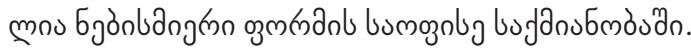

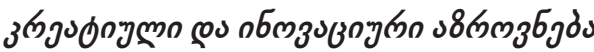

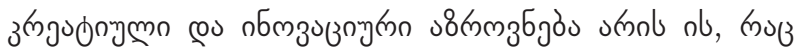

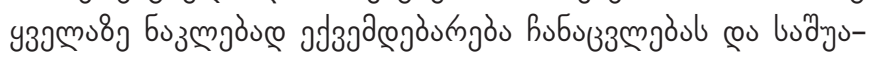

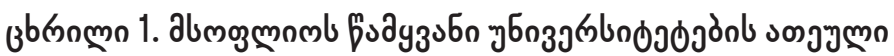

\begin{tabular}{|c|c|c|}
\hline 3m8nbns & 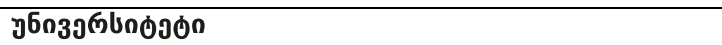 & 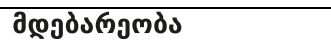 \\
\hline 1 & 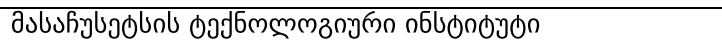 & دəֶ \\
\hline 2 & 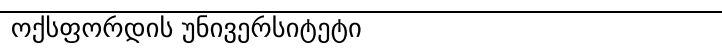 & 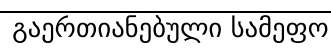 \\
\hline 3 & 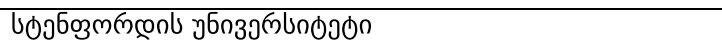 & נבֶ \\
\hline 3 & 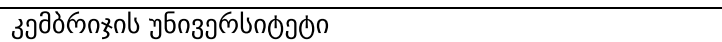 & 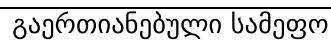 \\
\hline 5 & 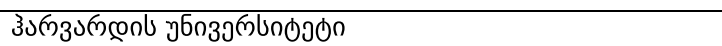 & נבy \\
\hline 6 & 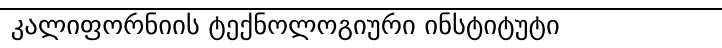 & ১Әे \\
\hline 7 & 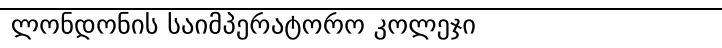 & 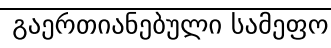 \\
\hline 8 & 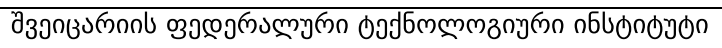 & $y_{30030}$ \\
\hline 8 & 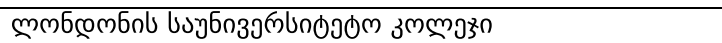 & 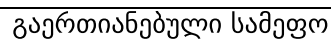 \\
\hline 10 & 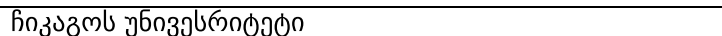 & गये \\
\hline
\end{tabular}

fyornm: www.topuniversities.com 


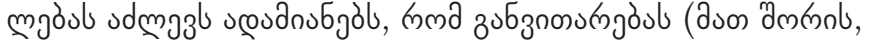

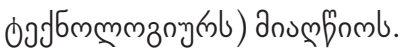

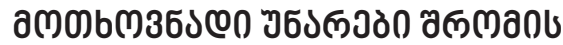

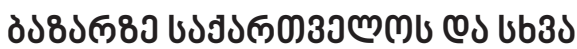

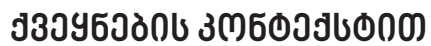

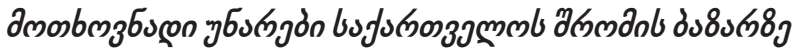

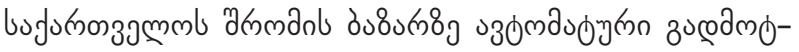

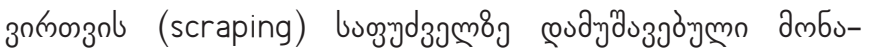

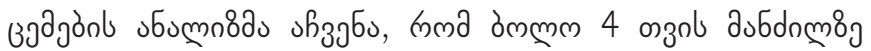

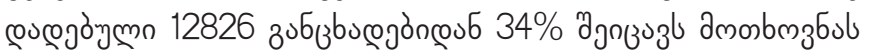

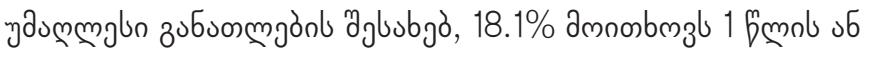

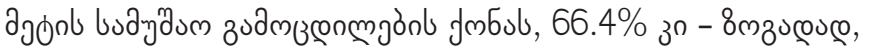

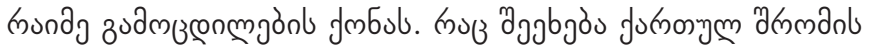

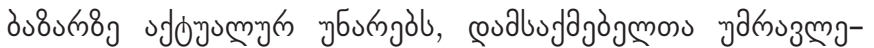

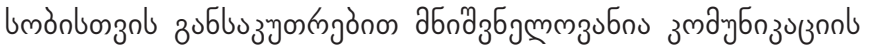
चб дмmbm

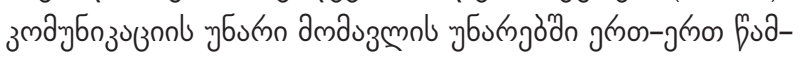

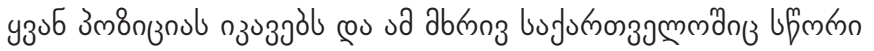

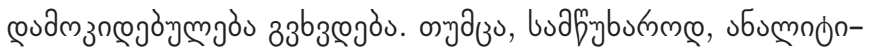

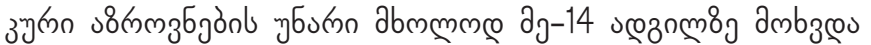

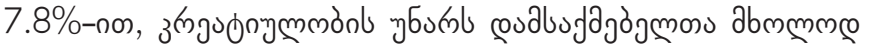

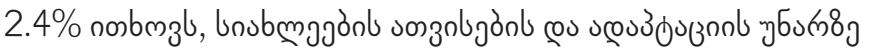

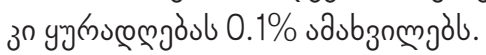

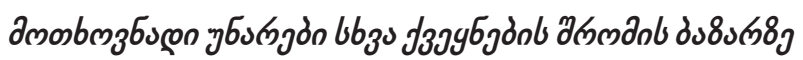

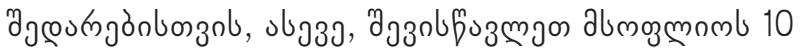

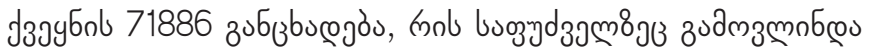

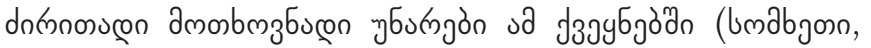

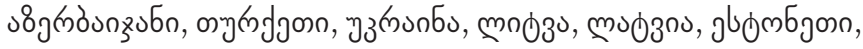
дмммкмз

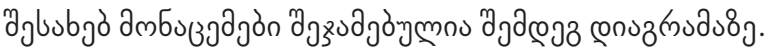

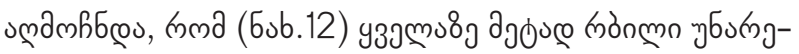

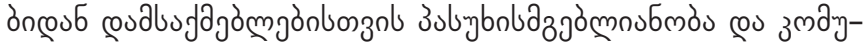

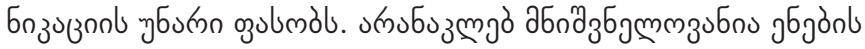

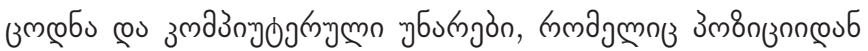

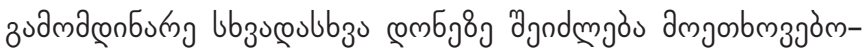

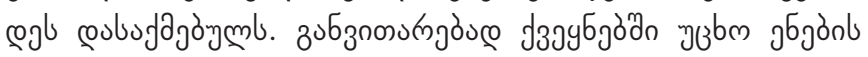

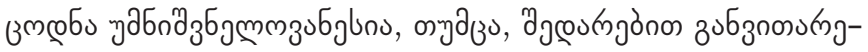

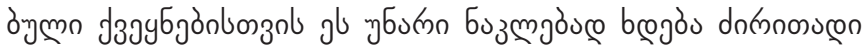

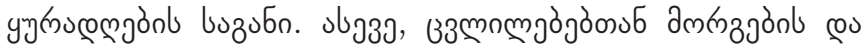

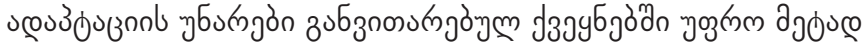
ogubmál.

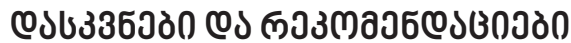

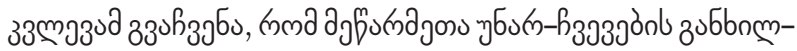

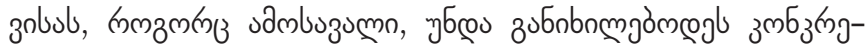

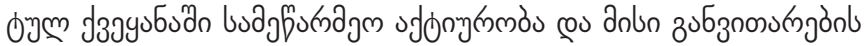

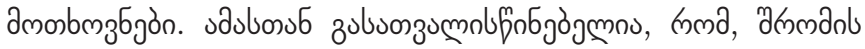

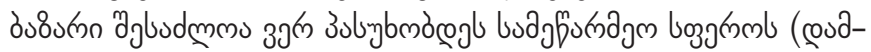

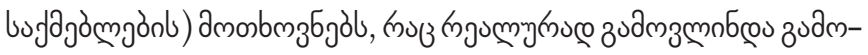

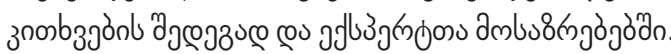

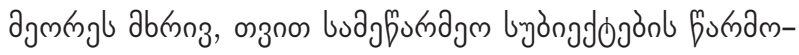

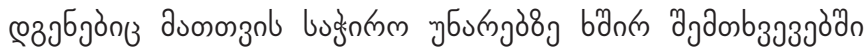

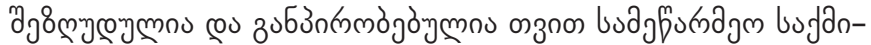

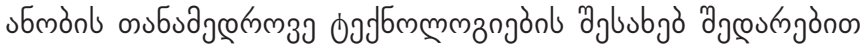

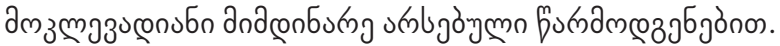

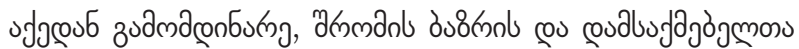

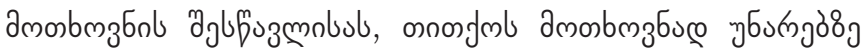

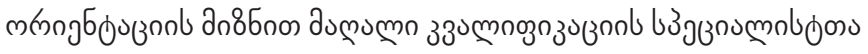

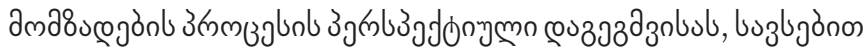

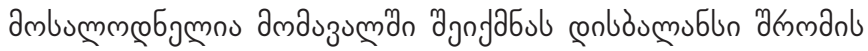

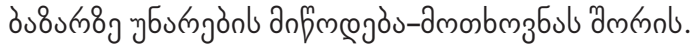

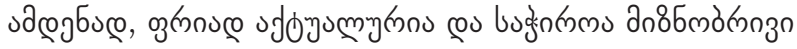

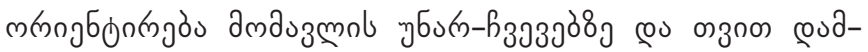

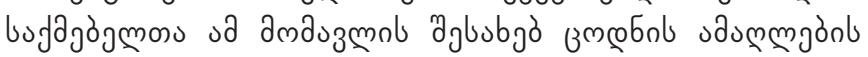

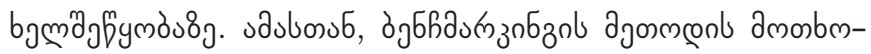

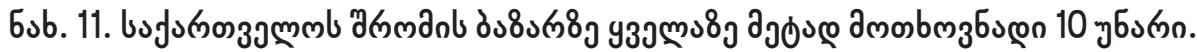

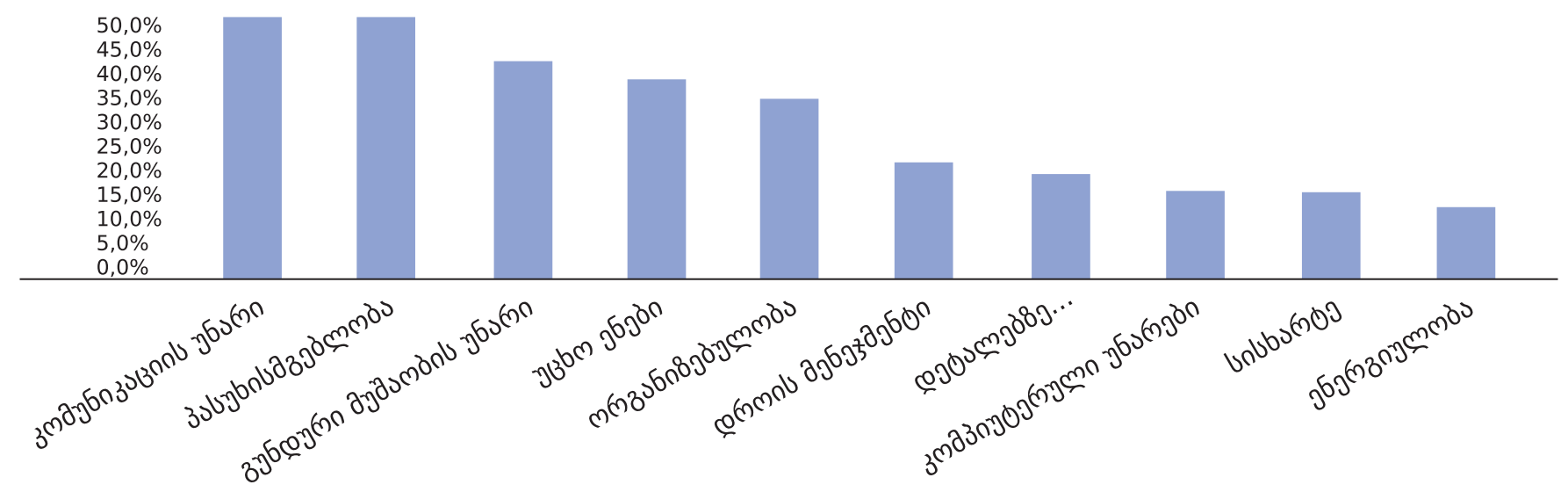




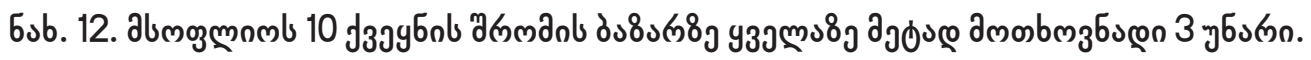

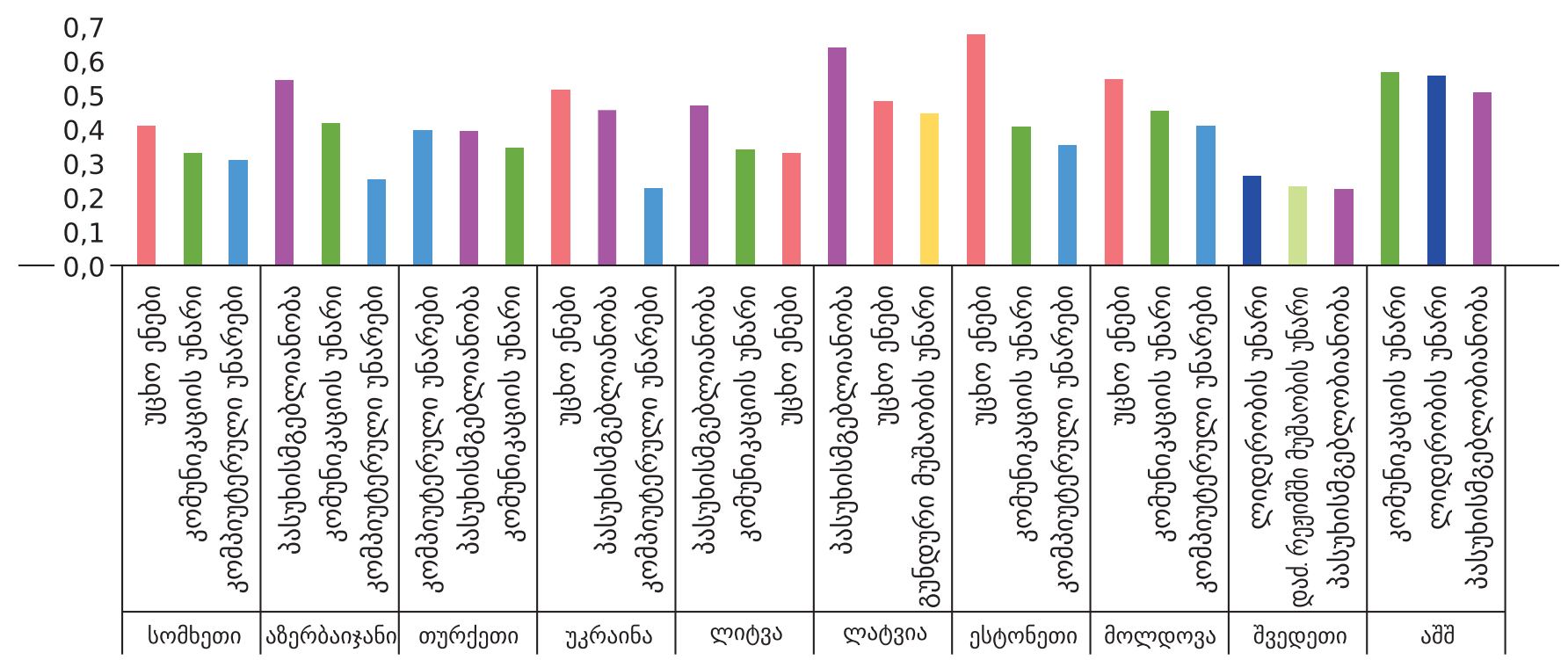

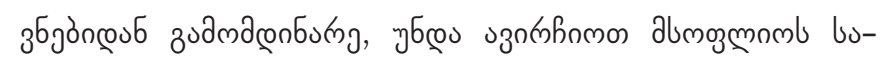

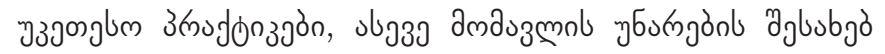

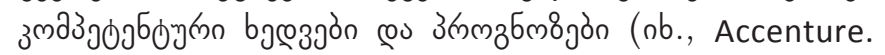
com\&NewSkills, 2016; Bakhshi et al., 2017; Bichia, 2017; 2018; 2020; Bughin et al, 2018; Dondi et al., 2021; Frey \& Osborne 2017; González-Velosa \& Rucci, 2016; Future Employment Growth..., 2020; Hugh Lauder \& Mayhew, 2020; McKinsey Global Institute, 2018; OECD, 2017; 2019; 2020; PMO Business Consulting, 2021; Raisch \& Krakowski, 2020; Stephanie, 2021; UKCES, 2014; World Economic Forum, 2016; 2018; Young et al., 2021).

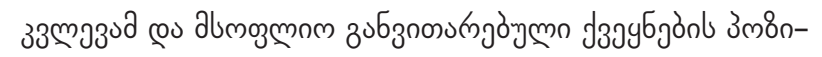

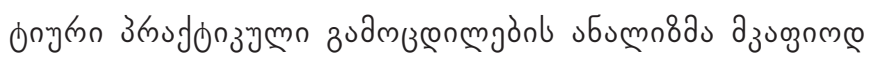

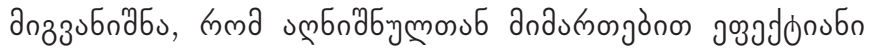

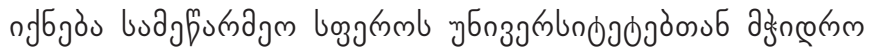

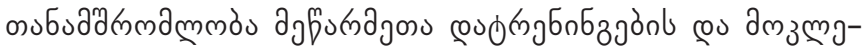

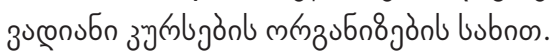

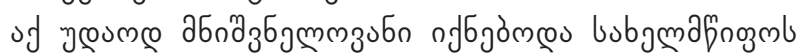

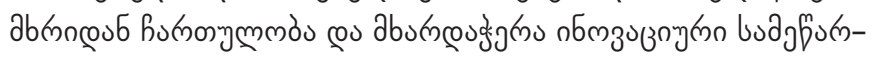

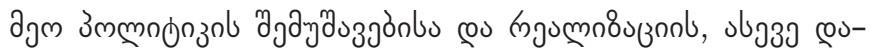

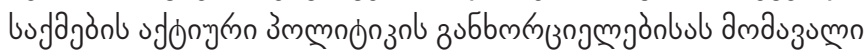

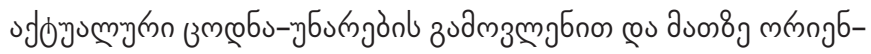

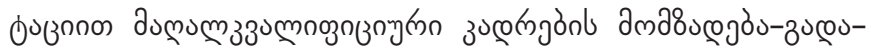

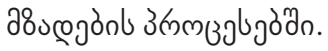

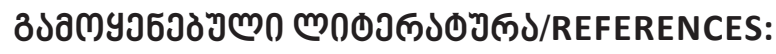

Accenture.com\&NewSkills (2016). New Skills Now: Inclusion in the Digital Economy.

Acemoglu, D., \& Restrepo, P. (2018). Low-Skill and High-Skill Automation. Journal of Human Capital, 12(2), $204-232$.

Acemoglu, D., \& Restrepo, P. (2019). Automation and New Tasks: How Technology Displaces and Reinstates Labor. Journal of Economic Perspectives, 33(2), 3-30.

Acs, Z. J., Szerb, L., \& Lloyd, A. (2018). Global Enterpreneurship Index. Washington, GEDI

Agrawal, S., De Smet, A., Poplawski, P. \& Reich, A. (2020). Beyond hiring: How companies are reskilling to address talent gaps. McKinsey \& Company.

Alaref, J., Brodmann, \& S., Premand, P. (2020). The Medium-Term Impact of Entrepreneurship Education on Labor Market Outcomes: Experimental Evidence from University Graduates in Tunisia. Labour Economics, 62, 101787. https://doi. org/10.1016/j.labeco.2019.101787

Alterbridge (2019). Compliance of the Quality of Higher Education in Georgia with the Requirements of the Local and International Labor Market. Tbilisi (In Georgian).

Amashukeli, M. (2021). Higher Education and the Labor Market - What Research Says (In Georgian).

Bakhshi, H., Downing, M. J. Osborne, A. M., \& Schneider, P. (2017). The Future of Skills: Employment in 2030.

Bedianashvili, G. (2017). Formation of Knowledge Economy and Innovative Entrepreneurial Policy: Institutional Aspects. Globalization and Business. 3, 10-16. (In Georgian). https://doi.org/10.35945/gb.2017.03.001

Bedianashvili, G. (2018). Culture as a Factor of Knowledge Economics with Paradigmatic Changes in Systemic Institutional Context. Globalization and Business. 6, 58-66 (In Georgian). https://doi.org/10.35945/gb.2018.06.007 
Bedianashvili, G. (2021). Macroeconomic and Cultural Determinants of the COVID-19 Pandemic Crisis. Bulletin of the Georgian National Academy of Sciences. 15 (2),191-197.

Bichia, Q. (2017). The Effect of Modern Globalization and Automatization Trend on Labor Market. The 2nd International Scientific Conference: Challenges of Globalization in Economics and Business. Ivane Javakhishvili Tbilisi State University Press, Tbilisi, 96-102. (In Georgian).

Bichia, Q. (2018). The Danger of Degradation of Education System and Labor Market in the Case of Misaligned Motivations. Globalization and Business. 6, 218-225. https://doi.org/10.35945/gb.2018.06.033

Bichia, Q. (2020). Accelerated Automation and the Ecosystem of Adaptation to the Changes. Globalization and Business, N10, 234-242. (In Georgian). https://doi.org/10.35945/gb.2020.10.030

Bughin, J., Hazan, E., Lund, S., Dahlström, P., Wiesinger, A., \& Subramaniam, A. (2018). Skill Shift Automation and the Future of the Workforce. McKinsey Global Institute.

Chanukvadze, N. (2021). Labor Market Analysis on the Example of Georgia (In Georgian).

Committee on Sectoral Economics and Economic Policy of the Parliament of Georgia (2018). Employment Problems and State Policy in the Field of Employment. Tbilisi (In Georgian).

Deloitte (2021). First Steps into the Labour Market 2021. International survey of students.

Diakonidze, A. (2018). Study of the Potential of Circular Migration of Georgian Labor Force in EU countries. Government Commission on Migration Issues. Tbilisi (In Georgian).

Dondi, M., Klier, J., Panier, F. \& Schubert, J. (2021). Defining the Skills Citizens Will Need in the Future World of Work. McKinsey \& Company.

Erkomaishvili, G. (2019). Digital Economy - an Innovative Tool for Economic Growth. Economics and Business, 2, $37-46$ (In Georgian).

Frey, C., B., Osborne \& M. A. (2017). The Future of Employment: How Susceptible Are Jobs to Computerisation? Technological Forecasting \& Social Change 114: 254-280.

Future employment growth (\% change) in EU27 in 2020-2030 across sectors (2020).

Gagnidze, I. (2018). About Some Directions of the Formation of an Entrepreneurial Ecosystem in Georgia. The 3rd International Scientific Conference: Challenges of Globalization in Economics and Business, Tbilisi, 77-81. (In Georgian).

Gagnidze, I. (2019). The Fourth Industrial Revolution - The Most Important Challenge of Higher Education in Georgia. Economics and Business, 3, 58-78. (In Georgian).

González-Velosa, C., Rucci, G. (2016). Methods to Anticipate Skills Demand.

Lauder, H., \& Mayhew, K. (2020). Higher Education and the Labour Market: an Introduction. Oxford Review of Education. 46(1), 1-19.

Khoshtaria, N. (2018). The Labor Market in Georgia Is Changing the Demands of not So Favorable Statistics (In Georgian).

Law of Georgia on Employment Promotion (2020). The parliament of Georgia (In Georgian).

McKinsey Global Institute. (2018). Skill Shift Automation and the Future of the Workforce.

Menshikov, V., Bedianashvili, G., Ruza, O., \& Kokina, I. (2021). Mobility in the Context of Entrepreneurial Potential of Students under the Conditions of the COVID-19 Pandemic (Latvia, Georgia). Journal of Entrepreneurship and Sustainability Issues. 9(1), 574-589. https://doi.org/10.9770/jesi.2021.9.1(36).

Ministry of Economy and Sustainable Development of Georgia (2017). Georgian labor market analysis. Tbilisi (In Georgian). Ministry of Economy and Sustainable Development of Georgia (2019). Georgian labor market analysis. Tbilisi (In Georgian). Ministry of Economy and Sustainable Development of Georgia (2020). Georgian labor market analysis. Tbilisi (In Georgian). OECD (2017), In-Depth Analysis of the Labour Market Relevance and Outcomes of Higher Education Systems: Analytical Framework and Country Practices Report, Enhancing Higher Education System Performance, OECD, Paris.

OECD (2019), OECD Employment Outlook 2019: The Future of Work, OECD Publishing, Paris.

OECD (2020), OECD Digital Economy Outlook 2020, OECD Publishing, Paris, https://doi.org/10.1787/bb167041-en.

Otarashvili, A. (2021). Active Employment Policy in the Labor Market (In Georgian).

PMO Business Consulting (2021). Research to Identify Labor Markets and Economic Sectors with Growth Potential.

Raisch, S., \& Krakowski, S. (2020). Artificial Intelligence and Management: The Automation-Augmentation Paradox. Academy of Management Review, (JA).

Stephanie, L. (2021). 9 Skills You'll Need to Succeed in a Post-Coronavirus Business World.

UKCES (2014). The Labour Market Story: Skills for the Future.

World Economic Forum. (2016). The Future of Jobs: Employment, Skills and Workforce Strategy for the Fourth Industrial Revolution.

World Economic Forum. (2018). The Future of Jobs: Centre for the New Economy and Society.

Young, J. S., Deller, M., F., \& Mccallum, E., K. (2021). Skills for the Post-Pandemic World: Innovation in Post-Secondary Education. PPF; Ted Rogers School of Management; Future Skills Centre. 